Fuzzy Cognitive Maps reasoning with words based on triangular fuzzy numbers Peer-reviewed author version

FRIAS DOMINGUEZ, Mabel; Filiberto, Yaima; NAPOLES RUIZ, Gonzalo; García-Socarrás, Yadira; VANHOOF, Koen \& Bello, Rafael (2017) Fuzzy Cognitive Maps reasoning with words based on triangular fuzzy numbers. In: Castro, Félix; Miranda-Jiménez, Sabino; González-Mendoza, Miguel (eds.). Advances in Soft Computing 16th Mexican International Conference on Artificial Intelligence, MICAI 2017, Enseneda, Mexico, October 23-28, 2017, Proceedings, Part I, p. 197-207..

DOI: $10.1007 / 978-3-030-02837-4 \_16$

Handle: http://hdl.handle.net/1942/25513 


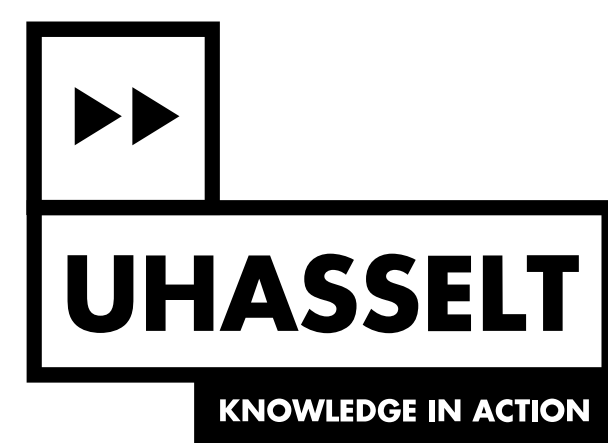

Fuzzy Cognitive Maps reasoning with words based on triangular fuzzy numbers Link

Peer-reviewed author version

Made available by Hasselt University Library in Document Server@UHasselt

Reference (Published version):

Frias, Mabel; Filiberto, Yaima; Nápoles, Gonzalo; García-Socarrás, Yadira; Vanhoof, Koen \& Bello, Rafael(2017) Fuzzy Cognitive Maps reasoning with words based on triangular fuzzy numbers. In: Proceedings of the 16th Mexican International Conference on Artificial Intelligence,

DOI: $10.1111 / j .1539-6924.2008 .01038 . x$

Handle: http://hdl.handle.net/1942/25513 


\title{
Fuzzy Cognitive Maps reasoning with words based on triangular fuzzy numbers
}

\author{
Mabel Frias ${ }^{1}$, Yaima Filiberto ${ }^{1}$, Gonzalo Nápoles ${ }^{2}$, \\ Yadira García-Socarrás ${ }^{4}$, Koen Vanhoof ${ }^{2}$, and Rafael Bello ${ }^{3}$ \\ 1 Universidad de Camagüey \\ Carretera de Circunvalación Norte entre Camino Viejo de Nuevitas y Ave Ignacio \\ Agramonte, Camagüey, Cuba

\begin{abstract}
A pivotal difference between Artificial Neural Networks and Fuzzy Cognitive Maps (FCMs) is that the latter allow modeling a physical system in terms of concepts and causal relations, thus equipping the network with interpretability features. However, such components are normally described by quantitative terms, which may be difficult to handle by domain experts. In this paper, we explore a reasoning mechanism for FCMs based on the Computing with Words paradigm where numerical concepts and relations are replaced with linguistic terms. More explicitly, we include triangular fuzzy numbers into the qualitative reasoning process attached to our model, thus proving further interpretability and transparency. The simulations show the potential behind the symbolic reasoning mechanism proposed in this study.
\end{abstract}

Keywords: Fuzzy cognitive maps, Computing with words, triangular fuzzy number, chondromalacia

\section{Introduction}

The reasoning mechanism behind Fuzzy Cognitive Maps (FCMs)[12] combines elements of fuzzy logic, neural networks and causal modeling. Fuzzy cognitive mapping allows modeling a real world system as a collection of concepts and causal relations [2]. One of the most attractive features attached to these knowledgebased networks lies in their graphical nature, their transparency and adaptability and their ability to perform WHAT-IF simulations. 
These advantages have motivated the scientific community to use fuzzy cognitive mapping in a wide spectrum of application domains including: social and political sciences, engineering, information technology, robotics, expert systems, education, prediction, environment, medicine, etc. Most of these solutions have a strong social and interdisciplinary scientific value as the study carried out by Nápoles et al. [15], who proposed an FCM-based representation of proteins for modeling the resistance of HIV mutations to existing drugs.

In an FCM, the knowledge is usually expressed by means of numerical values. However, in day-to-day activities, there are situations with imprecise information comprising qualitative aspects that are difficult to evaluate by the use of exact values [11]. Therefore, aiming at expanding the action field of fuzzy cognitive mapping, we combine its graphical nature with natural language techniques to describe both the concepts' activation values and the causal relations between them. In that way, we obtain a qualitative reasoning model.

In the FCM literature, some attempts to accomplish that can be found. For example, in [20] the authors proposed an FCM-model based on Computing with Words (CWW) [25] to improve the interpretability of diagnostic models of cardiovascular diseases. Gónzalez et al. [10] employed a representation model based on linguistic 2-tuple for modeling project portfolio interdependencies. Likewise, Rickard et al. [17] introduced another symbolic model based on interval type-2 fuzzy membership functions and the weighted power mean operator, $[8,9,18$, 19], while Dodurka et al. [6] analyzed the causal effect for fuzzy cognitive maps designed with non-singleton fuzzy numbers.

The use of linguistics terms to describe the whole network moves beyond the knowledge representation; preserving the semantics during the neural inference rule is pivotal towards developing an accurate linguistic model. In this paper, we further explore the hybridization between FCMs and the CWW paradigm where the activation vectors and the weight matrix are described using words. More precisely, we use triangular fuzzy numbers to describe the linguist terms. The numerical simulations using two case studies evidence the potentialities attached to our proposal when operating in qualitative scenarios.

The paper is organized as follows. Section 2 goes over some important concepts concerning to fuzzy cognitive mapping. In the Section 3, we provide a brief introduction to Computing with Words, whereas Section 4 describes the reasoning model for linguistic FCMs. The simulations are presented in the Section 5, while Section 6 provides concluding remarks.

\section{Fuzzy Cognitive Maps}

Fuzzy Cognitive Maps (FCMa) are a kind of recurrent artificial neural networks introduced by B. Kosko in 1986 [12]. These knowledge-based networks combine elements of fuzzy logic in their representation scheme and elements of neural networks to perform the inference process. Unlike classic neural networks that often operate like black-boxes, both concepts and relations in an FCM network have a precise meaning for the problem being modeled. 
The relationship between two concepts are characterized by a signed weight $w_{i j} \in[-1,1]$ that encloses a causal relationship [13]. The sign of $w_{i j}$ indicates whether the relationship between two concepts $C_{i}$ and $C_{j}$ is direct or inverse. These relationships have three possible states: i) if $w_{i j}>0$ then there is a positive causal relationship, thus suggesting that the first concept positively causes the second one; ii) if $w_{i j}<0$ then there is a negative causal relationship, which means that the first concept negatively causes the second one; and iii) if $w_{i j}=0$ then there is no causal relation between such concepts.

During the reasoning stage, an FCM-based model uses a neural reasoning rule to update the activation values of concepts given a certain activation vector. Equation 1 shows a widely used reasoning rule, where $N$ denotes the number of

concepts comprised into the causal network, $A_{j}^{(t)}$ denotes the activation value of the $C_{j}$ concept at the current iteration step, $w_{j i}$ is the causal weight to which $C_{j}$ causes $C_{i}$ and $f($.$) is a transfer function that maps the inner product into$ the activation interval e.g., $f(x)=1 /\left(1+e^{-\lambda x}\right)$.

$$
A_{i}^{(t+1)}=f\left(\sum_{j=1}^{N} A_{j}^{(t)} w_{j i}\right), i \neq j
$$

The above reasoning rule is repeated until either the network converges to a fixed-point attractor or a maximal number of cycles is reached. The former scenario implies that a hidden pattern was discovered [14] whereas the latter suggests that the system outputs are cyclic or chaotic. This iterative reasoning process allows performing WHAT-IF simulations through the modification of the activation values of meaningful processing entities.

\section{Computing with Words}

The introduction of the linguistic variable notion in 1973 by L. Zadeh opened new research horizons in the field of symbolic reasoning. This notion allows computing words instead of numbers [25] as an alternative to quantitative reasoning models. In general terms, the concept of linguistic variable is used to describe situations that are complex or are not clearly defined in quantitative terms. Moreover, the linguistic variables allow translating natural language into logical or numerical statements that can be effectively computed.

Long story short, Computing with Words (CWW) refers to the paradigm devoted to operating words or linguistic terms in order to build reasoning modules with high interpretability. The flexibility of CWW for modeling decision-making situations has boosted the emergence of several linguistic computational models. Some of these models are briefly described next.

- Linguistic Computational Model based on membership functions. The linguistic terms are expressed by fuzzy numbers, which are usually described by membership functions. This computational model makes the computations 
directly on the membership functions of the linguistic terms by using the Extension Principle [7].

$$
S^{n} \stackrel{\tilde{F}}{\rightarrow} F(R) \stackrel{a p p_{1}(.)}{\longrightarrow} S
$$

where $S^{n}$ symbolizes the $n$-Cartesian Product, $\tilde{F}$ is an aggregation operator based on the extension principle, $F(R)$ is the set of fuzzy sets over the set of real numbers and $\operatorname{app}_{1}($.$) is an approximation function that returns a label$ from the linguistic term set $S$.

- Linguistic Computational Symbolic Model [5]. This model performs the computation of indexes attached to linguistic terms. Usually, it imposes a linear order to the set of linguistic terms $S=\left\{S_{0}, \ldots, S_{g}\right\}$ where $S_{i}<S_{j}$ if and only if $i<j$. Formally, it can be expressed as:

$$
S^{n} \stackrel{R}{\rightarrow}[0, g] \stackrel{a p p_{2}(.)}{\longrightarrow}\{0, \ldots, g\} \rightarrow S
$$

where $R$ is a symbolic linguistic aggregation operator, $\operatorname{app}_{2}($.$) is an approx-$ imation function used to obtain an index $\{0, \ldots, g\}$ associated to a term in $S=\left\{S_{0}, \ldots, S_{g}\right\}$ from a value in the $[0, g]$ interval.

- The 2-tuple Fuzzy Linguistic Representation Model [11]. The above models perform simple operations with high transparency, but they have a common drawback: the loss of information caused by the need of expressing results in a discrete domain. The 2-tuple model is based on the notion of symbolic translation that allows expressing a domain of linguistic expressions as a continuous universe. This can be formalized as follows:

$$
S \stackrel{\Delta}{\rightarrow}\left(S_{i}, a_{i}\right) \stackrel{a p p_{3}(.)}{\longrightarrow}\left(S_{i}, \alpha_{i}\right) \stackrel{\Delta^{-1}}{\rightarrow} S
$$

where $S_{i} \in S$ and $\alpha_{i} \in[-0.5,0.5), \operatorname{app}_{3}($.$) is the aggregation operator for$ 2 -tuples, whereas the functions $\Delta$ and $\Delta^{-1}$ transform numerical values into a 2 -tuples and vice-versa, without losing information.

\section{FCM reasoning using Triangular Fuzzy Numbers}

In this section, we propose a linguistic FCM model that replaces the numerical components of the FCM reasoning (i.e., the concepts' activation values and the causal weights) with linguistic terms. In order to develop an effective linguistic FCM model, two key problems arise: (i) how to multiply two linguistic terms or words, and (ii) how to add the result of this product.

Let us consider the following set of linguistic terms: $S=\{V L /-V L$ (Very Low), $L /-L$ (Low), $M L /-M L$ (Moderate Low), $M /-M$ (Moderate), $M H /-$ $M H$ (Moderate High), $H /-H$ (High), $V H /-V H$ (Very High), $N A$ (No Activated)\}. The negative terms in $S$ will be used only to describe a negative causal weights $w_{i j}$ between two concepts since we are assuming that concept's activation values $C=\left\{C_{1}, C_{2}, \ldots, C_{N}\right\}$ are always positive. Figure 1 illustrates the membership functions associated with these terms. 


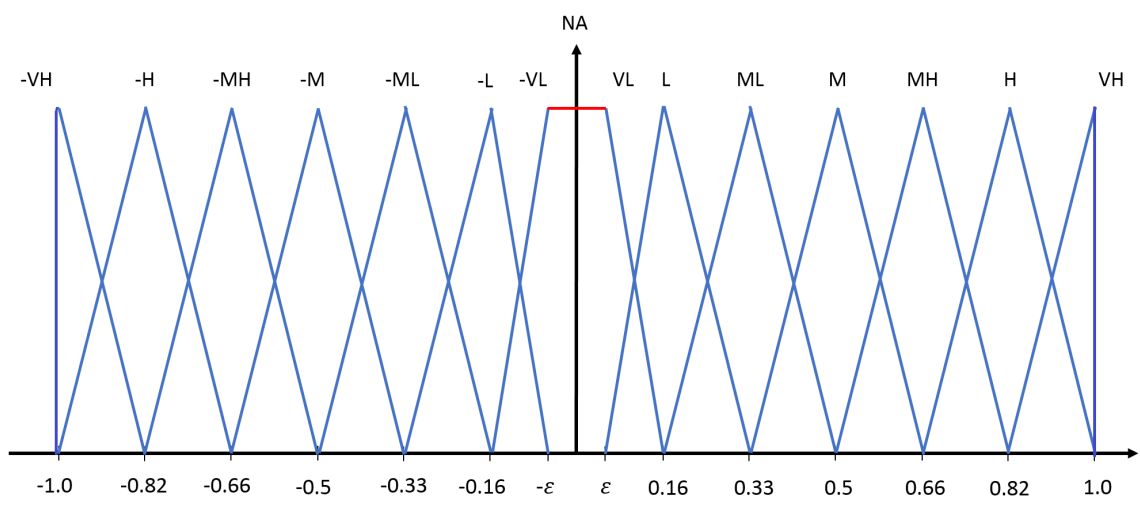

Fig. 1. Linguistic terms and their membership functions.

Let us consider a set of linguistic terms $S$ attached to $C_{i}$ and $w_{j i}$. Aiming at mapping the product $A_{j}^{(t)} w_{j i}$ into the CWW model, we consider the operator described in Equation 2, where $\tau\left(w_{j i}\right)$ and $\tau\left(A_{j}^{(t)}\right)$ are the triangular fuzzy numbers (TFN) [23] for $w_{i j}$ and $A_{i}^{(t)}$, respectively.

$$
I\left(w_{j i}, A_{j}^{(t)}\right)=\tau\left(w_{j i}\right) \tau\left(A_{j}^{(t)}\right)
$$

A triangular fuzzy number may be expressed as follows. Let $\hat{a}=\left[a^{L}, a^{M}, a^{U}\right]$, where $a^{L} \leq a^{M} \leq a^{U}$, then $\hat{a}$ is called a TFN, where $a^{L}$ and $a^{U}$ stand for the lower and upper values of $\hat{a}$, and $a^{M}$ is the modal value. There are many papers retaled to the fuzzy number arithmetic (e.g., [23, 24, 1,22]). In this paper, we adopted the notation introduced by [23] that defines the multiplication between two TFNs $\hat{a}=\left[a^{L}, a^{M}, a^{U}\right]$ and $\hat{b}=\left[b^{L}, b^{M}, b^{U}\right]$ as follows: $\hat{a} \times \hat{b}=\left[\min \left(a^{L} \times\right.\right.$ $\left.\left.b^{L}, a^{L} \times b^{U}, a^{U} \times b^{L}, a^{U} \times b^{U}\right), a^{M} \times b^{M}, \max \left(a^{L} \times b^{L}, a^{L} \times b^{U}, a^{U} \times b^{L}, a^{U} \times b^{U}\right)\right]$. Equation 3 displays the aggregation of the $N_{i}$ linguistic terms impacting the $i$ th concept, which produces a TFN codifying a linguistic term.

$$
\tau\left(C_{i}^{(t+1)}\right)=\sum_{j=1}^{N_{i}} I_{j}\left(w_{j i}, A_{j}^{(t)}\right)
$$

The next step of the proposed symbolic reasoning model for linguistic FCMbased systems is devoted to recovering the linguistic term attached to $\tau\left(C_{i}^{(t+1)}\right)$. With this goal in mind, we use the deviation between two TFNs as a distance function [4], which can be defined as follows:

$$
\delta(\hat{a}, \hat{b})=\sqrt{\frac{1}{3}\left[\left(a^{L}-b^{L}\right)^{2}+\left(a^{M}-b^{M}\right)^{2}+\left(a^{U}-b^{U}\right)^{2}\right]}
$$


Equation 5 displays the reasoning rule for this configuration, which computes the corresponding linguistic term for the $i$ th linguistic concept. This function determines the linguistic term reporting the minimal distance between its TFN and the one resulting from Equation 3. However, the linguistic term computed in this steps could be defined by a TFN comprising negative values, which is not allowed in our activation model. Aiming at overcoming this issue, we rely on a transfer function for symbolic domains.

$$
A_{i}^{(t+1)}=\underset{S_{k} \in S}{\arg \min }\left\{\delta\left(\tau\left(C_{i}^{(t+1)}\right), \tau\left(S_{k}\right)\right)\right\}
$$

Figure 2 shows the transfer function adopted in this paper, which is inspired on the sigmoid function. It should be highlighted that this function ensures computing positive linguistic values for concepts, while causal relations could be described using either positive or negative terms.

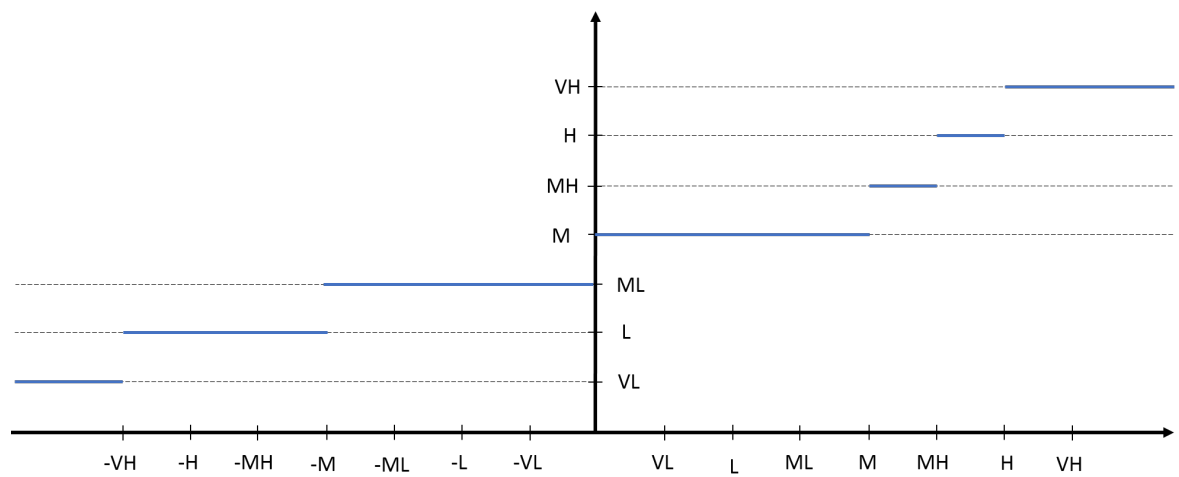

Fig. 2. Transfer function for symbolic domains

In order to show how the model operates, let us consider the FCM displayed in Figure 3. The activation values of concepts are fixed as follows: $C_{1} \leftarrow H i g h(H)$, $C_{2} \leftarrow \operatorname{High}(H), C_{3} \leftarrow \operatorname{Medium}(M), C_{4} \leftarrow \operatorname{Low}(L)$. The goal of this example is to compute the linguistic activation term for the $C_{5}$ concept.

Once the concepts have been activated, we can perform the reasoning process as explained above. This implies computing the linguistic activation value $A_{5}$ as the result of aggregating the linguistic activation terms attached to concepts $C_{1}-$ $C_{4}$ and their corresponding linguistic weights. Next we illustrate the operations related to one iteration in the symbolic reasoning process:

$$
\begin{gathered}
I_{1}=\tau(H) \tau(-H)=[0.66,0.82,1] *[-1,-0.82,-0.66]=[-1,-0.67,-0.44] \\
I_{2}=\tau(H) \tau(M)=[0.66,0.82,1] *[0.33,0.5,0.67]=[0.22,0.42,0.66]
\end{gathered}
$$




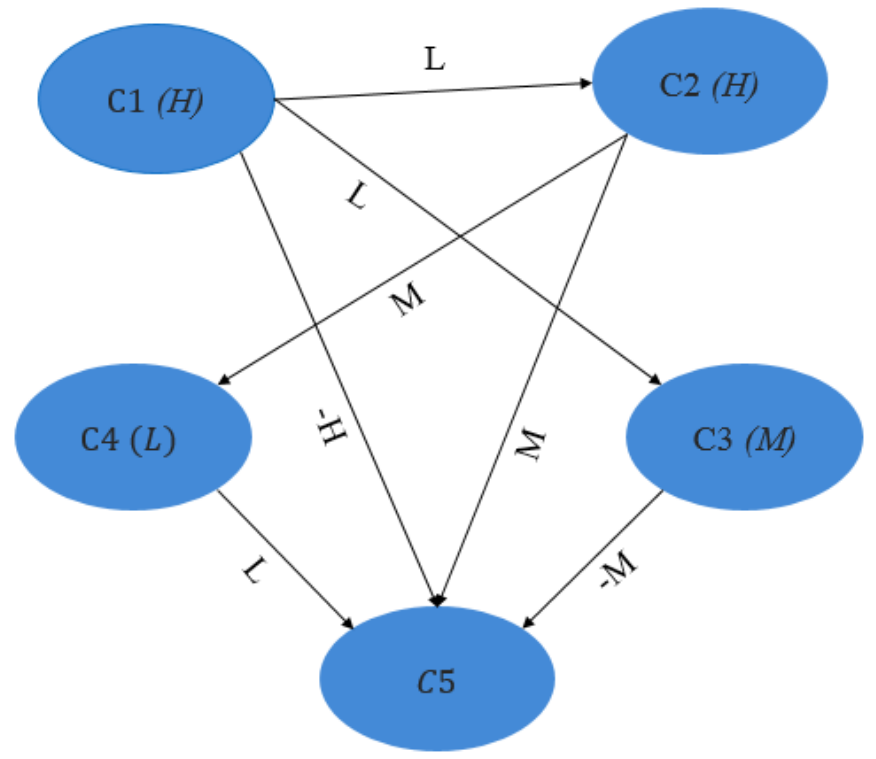

Fig. 3. Linguistic FCM-based system.

$$
I_{3}=\tau(M) \tau(-M)=[0.33,0.5,0.67] *[-0.66,-0.5,-0.33]=[-0.44,-0.25,-0.11]
$$

$$
I_{4}=\tau(L) \tau(L)=[0.01,0.16,0.33] *[0.01,0.16,0.33]=[0.0001,0.03,0.003]
$$

then,

$$
\begin{gathered}
\tau\left(C_{5}\right)=\left(I_{1}+I_{2}+I_{3}+I_{4}\right)=(-1,-0.48,0.11) \\
\delta\left(\tau\left(C_{5}\right), S_{1}\right)=\sqrt{\frac{1}{3}\left[(-1+1)^{2}+(-0.48+1)^{2}+(0.11+0.82)^{2}\right]}=0.62 \\
\vdots \\
\delta\left(\tau\left(C_{5}\right), S_{4}\right)=\sqrt{\frac{1}{3}\left[(-1+0.66)^{2}+(-0.48+0.5)^{2}+(0.11+0.33)^{2}\right]}=0.32
\end{gathered}
$$




$$
\begin{gathered}
\delta\left(\tau\left(C_{5}\right), S_{15}\right)=\sqrt{\frac{1}{3}\left[(-1+0.82)^{2}+(-0.48+1)^{2}+(0.11+1)^{2}\right]}=1.45 \\
A_{5}=\min \{0.62,0.49,0.38,0.32,0.34,0.56,0.64,0.64,0.65,
\end{gathered}
$$$$
0.70,0.85,1.01,1.16,1.32,1.45\}=0.32
$$$$
A_{5}=\underset{S_{k} \in S}{\arg \min }\left\{\delta\left(\tau\left(C_{i}^{(t+1)}, \tau\left(S_{k}\right)\right)\right\}=S_{4}=f(-M)=M L .\right.
$$

It is worth mentioning that our symbolic FCM-based model preserves its recurrent nature. This implies that the FCM will produce a state vector comprised of linguistic terms at each iteration-step until either a fixed-point is discovered or a maximal number of iterations is reached.

\section{$5 \quad$ Numerical simulations}

In this section, we present two case studies in order to asses the reliability of the proposed symbolic model for FCM-based systems.

\subsection{Crime and punishment}

Figure 4 displays the "crime and punishment" case study employed by Cavalho in [3] and Rickard in [17]. This FCM model attempts simulating the effects of various coupled social attributes on the prevalence of theft in a community. Aiming at illustrating how our model works for this case study, we need to transform the numerical weights into linguistic terms. Figure 1 portrays the triangular membership functions associated with the seven linguistic terms defined in $S$. These functions are regularly used in fuzzy cognitive modeling.

The experiments are oriented to calculating the linguistic activation values of each concept according to the proposed model using three simulation scenarios. These simulation scenarios are described as follow:

1. The concepts Community Intervention and Police Presence are set to $\mathrm{VH}$, while the others are set to $V L$. For this scenario, the symbolic FCM model converges in three iterations to the state vector:

$$
A=[M, M, M L, M, M, L, M H]
$$

whereas the linguistic FCM model proposed by Rickard in [17] converges in six iterations to a final state given by:

$$
B=[V H, M H, M L, V H, M, V H, V H] .
$$




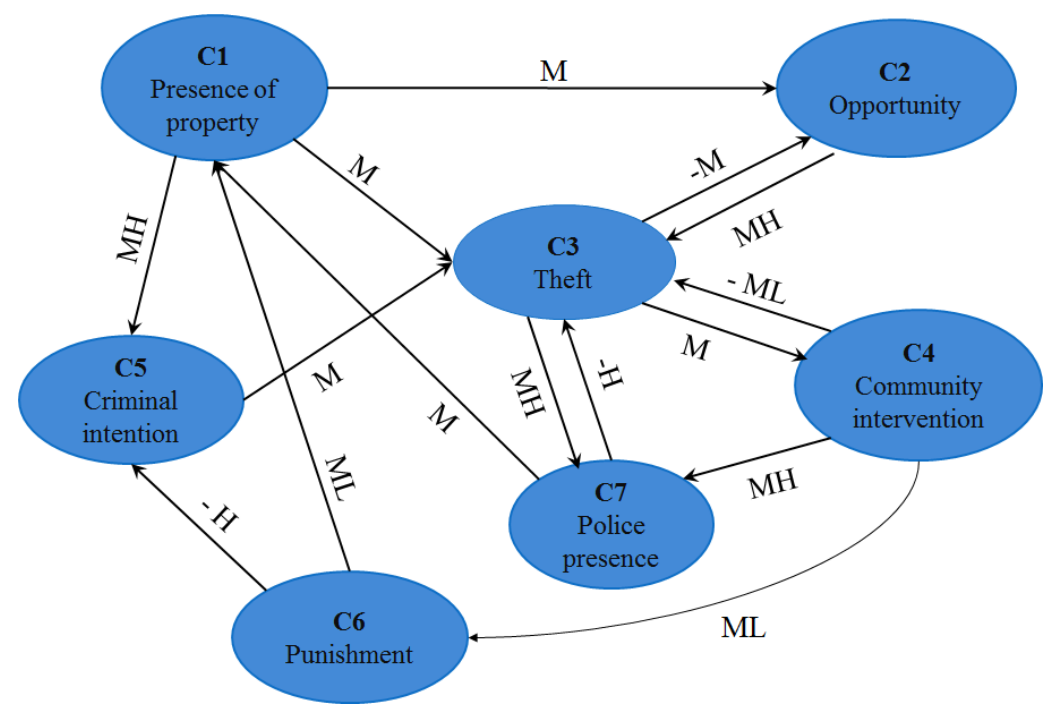

Fig. 4. Crime and punishment FCM model.

2. The concept Police Presence is set to $V H$, whereas the other map concepts are set to $V L$. For this second simulation scenario, the proposed linguistic FCM converges in three iteration-steps as well:

$$
A=[M, M, M L, M, M, L, M H]
$$

whereas Rickard's model converges in four iterations to the state:

$$
B=[H, M, M H, V L, H, V L, V H] .
$$

Notice that vectors are similar for key concepts. For example, the proposed linguistic FCM approach frequently converges to states where the activation value of Theft is lower than the activation value of Police Presence.

\subsection{Chondromalacia presence}

The second case study refers to a medical problem that analyzes the effects of different variables (i.e., concepts) leading to the presence of Chondromalacia in a patient. Chondromalacia patellae is a medical condition that affects the articular cartilage of the patella. It encompasses a spectrum of clinical severity, ranging from mild fissuring of the articular cartilage to complete cartilage loss and erosion of the underlying subchondral bone. Chondromalacia patellae can be considered a medical condition contained within the patellofemoral pain syndrome [16]. This medical condition has become an active reasearch field for practitioners. For example, Santiago-Santos et al. [21] evaluated the efficacy of hylan GF 20 administered immediately after arthroscopy. 
Using the opinion of three experts, we establish the causal relations between variables. Furthermore, we use two scenarios to analyze the activation values of concepts and validate the incidence of a concept to another. Figure 5 shows the linguistic FCM resulting from the experts' consensus.

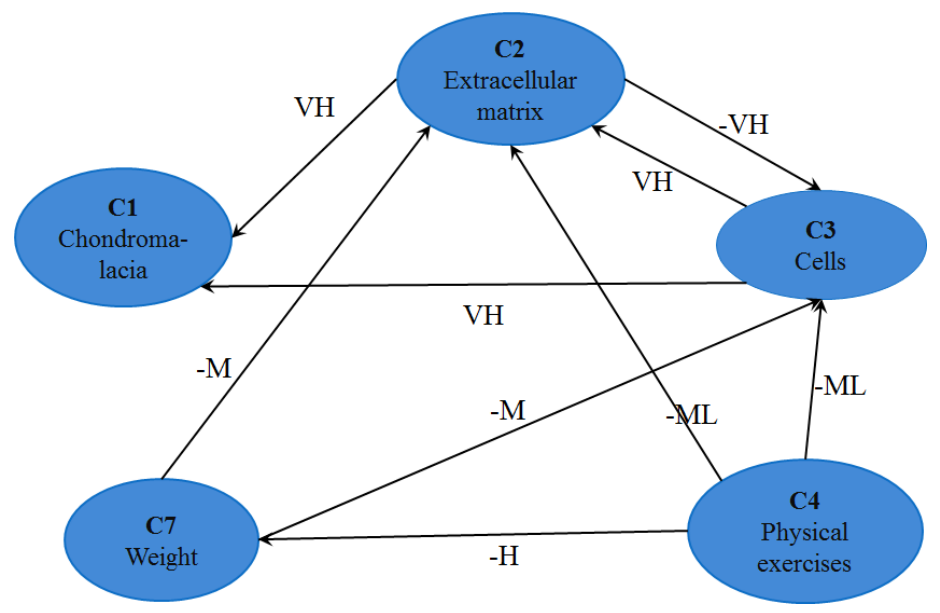

Fig. 5. Chondromalacia FCM model.

1. The concepts Extracellular matrix and Weight are set to $M$, while Chondromalacia is $M H, C e l l$ is $L$ and Physical exercises is $H$. For this scenario, the linguistic FCM converges to the following state vector:

$$
A=[M, L, L, H, L]
$$

2. The concept Chondromalacia is set to $M$, whereas the other concepts are set to $H$. For this second simulation scenario, the proposed linguistic model converges to the following state vector:

$$
A=[M, L, L, H, L]
$$

The results obtained are compatible with the observations made by experts who indicate that patients with low fissure of the articular cartilage, the presence of chondromalacia is medium. The criterion of experts referred to the need to carry out the study separately of each variable, was corroborated.

We have illustrated the practical advantages of using symbolic expressions to describe the FCM components and its reasoning mechanism, specially when performing WATH-IF simulations. As a result, we obtained a symbolic inference model with improved interpretability features, which is appreciated by users with no background in Mathematics or Computer Science. 


\section{Conclusions}

In this paper, we presented a symbolic reasoning mechanism for linguistic FCM models. In such systems, both the concepts' activation values and the causal weights are described by linguistic terms. In the proposed model, we referred to these terms as the linguistic activation values and the linguistic causal weights. Aiming at implementing this symbolic approach, we explored the use of triangular fuzzy numbers because of their simple interpretation.

The simulations have shown that our approach is able to computing consistent results. In spite of that, we observed differences on results when compared with the model proposed by Rickard et al [17]. We could conjecture that these differences may be a result of losing relevant information when processing information on such qualitative models. The lack of flexibility on the definition of triangular fuzzy numbers may also affect the model's accuracy. These issues, however, become a strong motivation towards exploring other alternatives to improve the performance of our model as a future research work.

\section{Acknowledgment}

The authors would like to thank Isel Grau (Vrije Universiteit Brussel, Belgium) for her valuable suggestions on the transfer function design.

\section{References}

1. Akther, Thowhida Ahmad, S.U.: A computational method for fuzzy arithmetic operations. Daffodil international university journal of science and technology 4(1), 18-22 (2009)

2. Bourgani, E., Stylios, C.D., Georgopoulos, Voula C.and Manis, G.: A study on fuzzy cognitive map structures for medical decision support systems. In: 8th Conference of the European Society for Fuzzy Logic and Technology (EUSFLAT 2013) (2013)

3. Carvalho, J.: On the semantics and the use of fuzzy cognitive maps and dynamic cognitive maps in social sciences. Fuzzy Sets \& Syst 214, 6-19 (2013)

4. Chen, C.: Extension of the topsis for group decision-making under fuzzy environment. Fuzzy Sets and Systems 114, 1-9 (2000)

5. Delgado, M., Verdegay, J.L., Vila, M.A.: On aggregation operations of linguistic labels. Int. J. Intell. Syst (8), 351-370 (1993)

6. Dodurka, M.F., Yesil, E., Urbasa, L.: Causal effect analysis for fuzzy cognitive maps designed with non-singleton fuzzy numbers. Neurocomputing 232, 122-132 (2017)

7. Dubois, D., Prade, H.: Fuzzy Sets and Systems: Theory and Applications. New York: Academic (1980)

8. Dujmovic, J.: Continuous preference logic for system evaluation. IEEE Trans. Fuzzy Syst 15(6), 1082-1099 (2007)

9. Dujmovic, J., Larsen, H.: Generalized conjunction/disjunction. Int. J. Approximate Reasoning. 46, 423-446 (2007) 
10. Gónzalez, M.P.n., Rosa, C.G.B.D.L., Moran, F.J.C.n.: Fuzzy cognitive maps and computing with words for modeling project portfolio risks interdependencies. International Journal of Innovation and Applied Studies 15(4), 737-742 (mayo, 2016)

11. Herrera, F., Martínez, L.: A 2-tuple fuzzy linguistic representation model for computing with words. IEEE Transactions on Fuzzy Systems 8(6), 746-752 (2000)

12. Kosko, B.: Fuzzy cognitive maps. International Journal of Man-Machine Studies 24, 65-75 (1986)

13. Kosko, B.: Neural Networks and Fuzzy Systems, a Dynamic System Approach to Machine Intelligence. Englewood Cliffs (1992)

14. Kosko, B.: Hidden patterns in combined and adaptive knowledge networks. International Journal of Approximate Reasoning 2(4), 377 - 393 (1988)

15. Nápoles, G., Grau, I., Bello, R., Grau, R.: Two-steps learning of fuzzy cognitive maps for prediction and knowledge discovery on the HIV-1 drug resistance. Expert Systems with Applications 41(3), 821-830 (2014)

16. Ramírez Sánchez, K.T.: Condromalacia rutinaria. Revista Médica de Costa Rica y Centroamerica LXXI, 551 - 553 (2014)

17. Rickard, J.T., Aisbett, J., Yager, R.R.: Computing with words in fuzzy cognitive maps (2015)

18. Rickard, J., Aisbett, J., Yager, R., Gibbon, G.: Fuzzy weighted power means in evaluation decisions (2010)

19. Rickard, J., Aisbett, J., Yager, R., Gibbon, G.: Linguistic weighted power means: comparison with the linguistic weighted average (2011)

20. Saleh, S.H., Rivas, S.D.L., Gomez, A.M.M., Mohsen, F.S., Vzquez, M.L.: Representación del conocimiento mediante mapas cognitivos difusos y conjuntos de términos lingüisticos difusos dudosos en la biomedicina. International Journal of Innovation and Applied Studies 17(1), 312-319 (2016)

21. Santiago-Santos A, Blancas-Vargas ME, S.E.J.: Eficacia del hilano g-f 20 aplicado en dosis única intraarticular posterior a artroscopia en el tratamiento del sndrome de dolor patelo-femoral. Rev Sanid Milit Mex 69, 301-308 (2015)

22. Su, W., Peng, W., Zeng, S., Pen, B., Pand, T.: A method for fuzzy group decision making based on induced aggregation operators and euclidean distance. International transactions in operational research 20, 579594 (2013)

23. Van, L., Pedrycz, W.: A fuzzy extension of saatys priority theory. Fuzzy Sets and Systems 11, 229241 (1983)

24. Xu, Z.: Fuzzy harmonic mean operators. International Journal of Intelligent Systems 24, $152172(2009)$

25. Zadeh, L.A.: Outline of a new approach to the analysis of complex systems ad decision processes. IEEE Transactions Systems, Man, and Cybernetics SMC-3(1), 28-44 (January,1973) 